\title{
Antimicrobial Resistance of Escherichia coli Causing Uncomplicated Urinary Tract Infections: A European Update for 2014 and Comparison with 2000 and 2008
}

Gunnar Kahlmeter · Jenny Åhman · Erika Matuschek

To view enhanced content go to www.infectiousdiseases-open.com

Received: September 2, 2015 / Published online: October 27, 2015

(C) The Author(s) 2015. This article is published with open access at Springerlink.com

\section{ABSTRACT}

Introduction: The objective of this study was to provide an update on the resistance of Escherichia coli in women with acute uncomplicated urinary tract infections (UTIs) in France, Germany, Spain, Sweden, and the United Kingdom (UK) to mecillinam [amdinocillin (United States Adopted Name)], amoxicillin-clavulanic acid, cefadroxil, nitrofurantoin, ciprofloxacin, and trimethoprim, and to compare the results with resistance in the ECO.SENS I and II surveys in 2000 and 2008, respectively.

Methods: The susceptibility of $E$. coli in France (166 isolates), Germany (133 isolates), Spain (169 isolates), Sweden (137 isolates), and the UK (124 isolates) was determined by disc diffusion according to European Committee on Antimicrobial Susceptibility Testing (EUCAST)

Electronic supplementary material The online version of this article (doi:10.1007/s40121-015-0095-5) contains supplementary material, which is available to authorized users.

G. Kahlmeter $(\bowtie) \cdot J$. Åhman · E. Matuschek

Department of Clinical Microbiology, Central

Hospital, Växjö, Sweden

e-mail: gunnar.kahlmeter@kronoberg.se breakpoints and methodology. Resistance rates were compared using Fisher's exact test, 2-tailed, with $P<0.05$ indicating statistical significance.

Results: Since 2000, there has been a significant increase in resistance to cefadroxil in Germany (1\% to $12 \%$ ) and Spain (3\% to $8 \%$ ), to ciprofloxacin in Germany (2\% to $21 \%$ ), Spain (15\% to $31 \%)$, Sweden ( $0 \%$ to $7 \%$ ), and the UK ( $1 \%$ to $15 \%)$, to trimethoprim in Germany (23\% to $37 \%)$, Spain (25\% to $37 \%$ ), Sweden (9\% to $17 \%)$, and the UK (13\% to $46 \%)$, to mecillinam in Spain (1\% to $6.5 \%)$, and to nitrofurantoin in the UK ( $0 \%$ to $6 \%)$; there was also a significant decrease in resistance to nitrofurantoin in Spain (4\% to $0 \%$ ). Since 2008, there has been a significant increase in resistance to ciprofloxacin in Sweden (3\% to 15\%) and the UK ( $1 \%$ to $15 \%)$, and to trimethoprim (13\% to $46 \%$ ) and nitrofurantoin ( $0 \%$ to $6 \%$ ) in the UK. Conclusion: E. coli isolates from women with acute uncomplicated UTIs have increasing antimicrobial resistance, particularly to ciprofloxacin and trimethoprim. However, resistance to mecillinam and nitrofurantoin mostly remains low.

Funding: LEO Pharma. 
Keywords: Amoxicillin-clavulanic

acid; Antimicrobial resistance; Susceptibility; Cefadroxil; Ciprofloxacin; Escherichia coli; Mecillinam; Nitrofurantoin; Trimethoprim; Urinary isolates; Urinary tract infections

\section{INTRODUCTION}

Antimicrobial resistance (AMR) in clinically important pathogens, such as Escherichia coli, is of continuing concern [1]. Changes in resistance levels are best monitored by prospective, multi-national, surveillance programs, employing standardized procedures to collect the isolates and determine their antimicrobial susceptibility [2]. The ECO.SENS (The pan-European Escherichia coli sensitivity survey) project was the first such study to investigate the antimicrobial susceptibility of E. coli in acute uncomplicated urinary tract infections (UTIs) in women. It was first conducted between 1999 and 2000, involving 1417 isolates from 4734 women in 17 countries [3], and again in 2008 involving 903 isolates from 1697 women in 5 countries [4].

We report here an update on the antimicrobial susceptibility of isolates of similar origin from five countries which participated in either both or one of the previous surveys representing areas of Europe with varying AMR problems.

\section{METHODS}

The selection of patients and procedures involved has been reported previously and are summarized only briefly here [3, 4]. Methodological differences if any are described. The study involved isolates of $E$. coli from non-hospitalized females aged 18 years and over, with symptoms of acute uncomplicated lower UTI, which included any or all of the following: frequency, dysuria, urgency, and suprapubic pain. In France (14 centers), Spain (3 centers), Sweden (1 center), and the United Kingdom (UK; 2 centers), all isolates were from patients in primary healthcare. In Germany (four centers), isolates were from women attending hospital clinics and the laboratory had difficulties guaranteeing the community origin of the isolates. Isolates were sent to the Department of Clinical Microbiology at the Central Hospital, Växjö, Sweden. Their antimicrobial susceptibility to mecillinam [amdinocillin (United States Adopted Name)], amoxicillin-clavulanic acid, cefadroxil (as a screen for cephalosporin resistance, particularly ESBL production), nitrofurantoin, ciprofloxacin, and trimethoprim was determined by disc diffusion using the European Committee on Antimicrobial Susceptibility Testing (EUCAST) methodology and breakpoints [5, 6]. Isolates resistant to the first-generation cephalosporin, cefadroxil, were investigated for production of extended-spectrum beta-lactamase (ESBL) by double disc synergy testing using cefotaxime with and without clavulanic acid and ceftazidime with and without clavulanic acid.

Resistance rates were compared to those obtained in 2000, for all countries and to those obtained in 2008 for Sweden and the UK for all antimicrobials with the exception of amoxicillin-clavulanic acid. The methodology for determining susceptibility to amoxicillin-clavulanic acid in the current study was different from that used in the previous studies [7]. Statistical comparison was by Fisher's exact test, 2-tailed, with $P<0.05$ indicating statistical significance.

All procedures followed were in accordance with the ethical standards of the responsible committee on human experimentation at the 
respective institutions and with the Helsinki Declaration of 1964, as revised in 2013. No patient identifiers other than sex and age were recorded and, as such, informed consent was not required for inclusion in this study.

\section{RESULTS}

Percentage susceptibilities to the agents tested are shown in Table 1. When compared to the results from 2000, there were instances where resistance had increased significantly in 2014, as follows: Germany showed a significant increase in resistance to cefadroxil $(1.4 \%$ to $12.0 \% ; \quad P<0.001)$, ciprofloxacin $(2.2 \%$ to $20.2 \% ; P<0.0001)$, and trimethoprim $(22.5 \%$ to $36.8 \% ; P<0.05$ ); Spain showed a significant increase in resistance to mecillinam $(1.0 \%$ to $6.5 \% ; \quad P<0.01)$, cefadroxil $(3.1 \%$ to $8.3 \%$; $P<0.05), \quad$ ciprofloxacin $\quad(14.7 \%$ to $30.8 \%$; $P<0.001)$, and trimethoprim $(25.1 \%$ to $37.3 \%$; $P<0.05)$; Sweden showed a significant increase in resistance to ciprofloxacin $(0 \%$ to $7.3 \%$; $P<0.001)$ and trimethoprim $(8.8 \%$ to $16.9 \%$; $P<0.05)$; and the UK showed a significant increase in resistance to ciprofloxacin $(0.6 \%$ to $15.3 \% ; \quad P<0.0001)$, trimethoprim $(13.3 \%$ to $46.0 \% ; P<0.0001)$, and nitrofurantoin $(0 \%$ to 5.6\%; $P<0.01)$. Spain also showed a significant decrease in resistance to nitrofurantoin $(4.2 \%$ to $0 \% ; P<0.01)$.

Resistance in 2014 was significantly greater than that recorded in 2008 in the UK with a significant increase in resistance to ciprofloxacin $(0.5 \%$ to $15.3 \% ; \quad P<0.0001)$, nitrofurantoin $(0 \%$ to $5.6 \% ; P<0.01)$, and trimethoprim ( $14.9 \%$ to $46.0 \% ; P<0.0001)$.

Overall there were $35(4.8 \%)$ isolates positive for ESBL production. The incidence in each country was $2.4 \%$ in France, $10.5 \%$ in Germany,
$4.7 \%$ in Spain, $2.9 \%$ in Sweden, and $4.0 \%$ in the UK.

\section{DISCUSSION}

E. coli from women with acute uncomplicated UTIs in 2014 showed high levels of resistance to ciprofloxacin, trimethoprim, and amoxicillin-clavulanic acid. Significantly greater resistance when compared to similar isolates from both 2000 and 2008 was noted for ciprofloxacin and trimethoprim. The current study is particularly important in that it enables trends in resistance to be compared over a 15 -year period, as opposed to the usual surveys which take only a snapshot at a specific time. When compared to 2000, ciprofloxacin and trimethoprim resistance had increased in Germany, Spain, Sweden, and the UK. Resistance had similarly increased to ciprofloxacin and trimethoprim in the UK since 2008.

There were only minor differences in methodology between the first two surveys and that reported here. The surveys in 2000 and 2008 involved many centers in each country $[3,4]$. In contrast, the latest study was truly multi-center only in France, whereas samples from the other countries came from up to four centers. No upper age limit was imposed on patients in the current study, whereas an upper age limit of 65 years was specified previously. Despite these differences, the three surveys had very important similarities. All involved women with acute uncomplicated UTIs. Urine samples in all three surveys were obtained from women in primary healthcare, with the exception of Germany in the current study, where most of the isolates were from women attending 


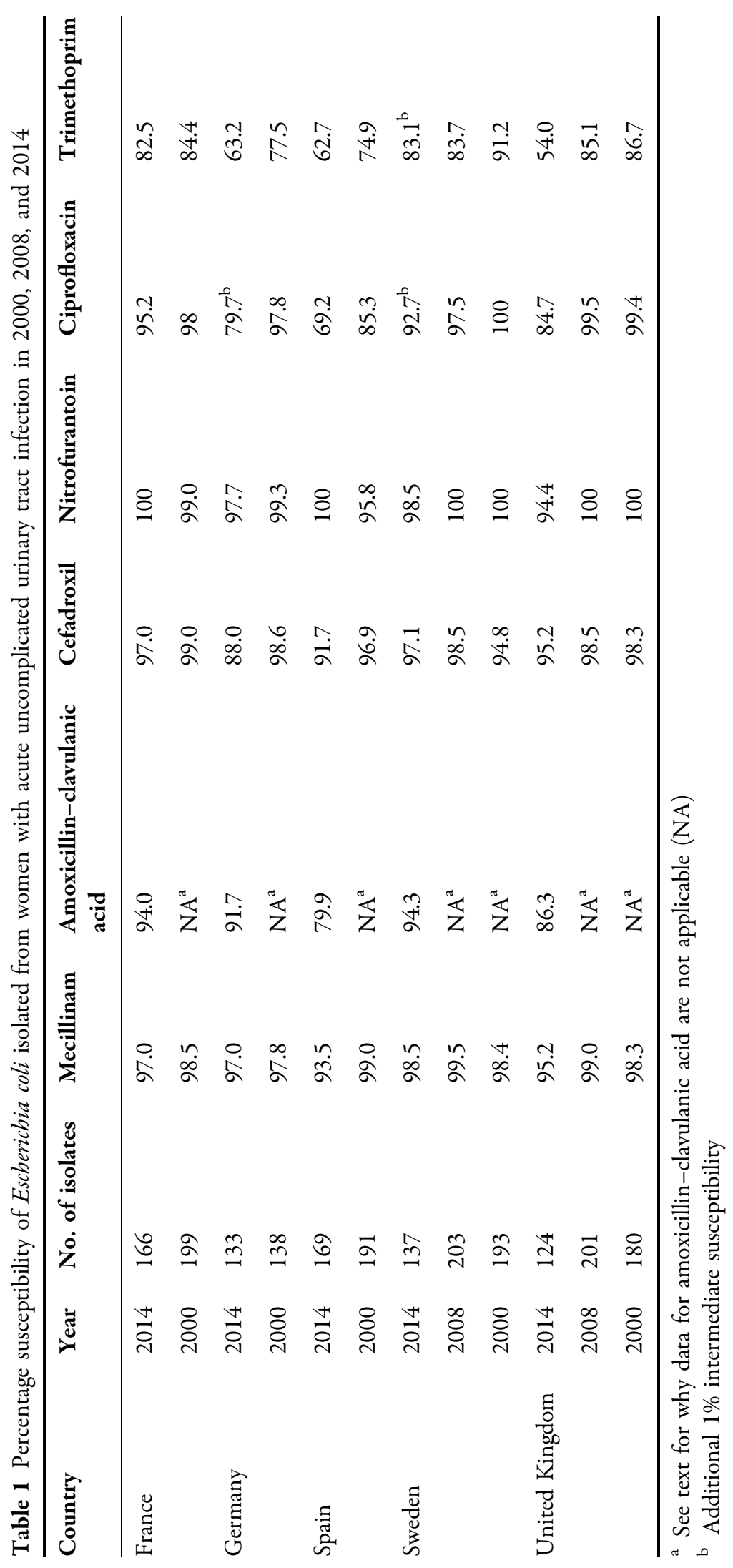


hospital clinics. Antimicrobial susceptibility was performed at the same laboratory for all three studies.

Susceptibility in the current study was determined using EUCAST breakpoints and standardized methodology $[5,6]$. The methodology used in the current study was different to that used previously, as it used a different medium and inoculum as specified by EUCAST. However, the zone diameter breakpoints were the same for all antimicrobials with the exception of amoxicillin-clavulanic acid [5-7]. The most recent guidelines for amoxicillin-clavulanic acid use a fixed concentration of clavulanic acid and apply specifically to urinary isolates [5, 6]. The new susceptibility breakpoint for isolates from UTIs is defined as an amoxicillin minimum inhibitory concentration of $\leq 32 \mathrm{mg} / \mathrm{L}$. Due to these differences in methodology it is impossible and inappropriate to compare the resistance rates for this antimicrobial between on one hand 2014 and on the other 2000 and 2008. However, it is acceptable to compare the results from the three surveys for all other antimicrobials. Statistical comparison is also acceptable, although for the reasons discussed above, one should not place too much emphasis upon the degrees of statistical significance found.

Our results and the trends observed are comparable to those observed elsewhere over a similar timescale. In France, resistance to amoxicillin-clavulanic acid [8] and trimethoprim-sulfamethoxazole $[8,9]$ has been highlighted, and there are similar reports from Germany [10, 11], along with resistance to cephalosporins [10]. Resistance to quinolones and trimethoprim-sulfamethoxazole in Spain is well documented [12, 13]. Although antimicrobial susceptibility in E. coli isolates in Sweden is generally high, trimethoprim resistance levels have been a cause for concern [14]. Similarly, the high level of trimethoprim resistance in the UK is well documented [15].

The Antimicrobial Resistance Epidemiological Survey on Cystitis (ARESC) study in Europe and Brazil, which involved similar patients to those in our study, noted that susceptibility to E. coli of over $90 \%$ was found for mecillinam, nitrofurantoin, and fosfomycin only [16]. Although fosfomycin was not tested in our latest study, consistent and high susceptibility of $E$. coli in all countries was similarly noted for mecillinam and nitrofurantoin. Interestingly, investigation of 34 isolates of $E$. coli resistant to either mecillinam or nitrofurantoin from the first ECO.SENS study in 2000 indicated the low probability of future clonal spread of resistance to these agents [17]. Our update has confirmed no major increase in mecillinam or nitrofurantoin resistance has taken place in the last 15 years.

ESBL production was recorded in 35 (4.8\%) isolates. This is greater than that recorded previously in the 2008 ECO.SENS study (1.2\% of isolates), suggesting that the frequency of ESBL-producing E. coli in the community is increasing. The incidence was less than $5 \%$ in all countries except Germany, where it was $10.5 \%$, where isolates were from women attending hospital clinics.

The continuing and increasing resistance to ciprofloxacin and trimethoprim is disturbing and impacts on the choice of therapy for women with acute uncomplicated UTIs, particularly in domiciliary practice, where treatment is usually empirical. Antimicrobial susceptibility surveys such as the ECO.SENS and ARESC are essential and provide the most appropriate information on which to base such treatment. Our data, and that of others, indicate that mecillinam, nitrofurantoin, and 
fosfomycin are suitable options for empirical therapy of women with acute uncomplicated UTI. These are old agents with indications limited to lower uncomplicated UTIs. Interest in the redevelopment of these old agents has awakened with the galloping resistance to other agents and with the lack of new agents [18].

\section{CONCLUSIONS}

E. coli isolates from women with acute uncomplicated UTIs showed a significant increase in AMR since 2000 to ciprofloxacin and trimethoprim (Germany, Spain, Sweden, and the UK). Susceptibility to mecillinam and nitrofurantoin remains high. The ECO.SENS and ARESC studies indicate that suitable agents for empirical treatment of acute cystitis include mecillinam, nitrofurantoin, and fosfomycin.

\section{ACKNOWLEDGMENTS}

The article processing charges for this study were funded by Leo Pharma, Denmark. Colleagues Jordi Vila, Rafael Canton and Luis Martinez-Martinez (Spain), Sören Gatermann (Germany), Karen Bowker and Mandy Wootton (UK), and Vincent Jarlier (France) are thanked for organizing the collection of isolates in the respective countries. This material was presented in part at the 25th European Congress of Clinical Microbiology and Infectious Disease, Copenhagen, Denmark, April 25-28, 2015. The assistance of Paul Menday in the statistical analysis and preparation of this manuscript is acknowledged. All named authors meet the International Committee of Medical Journal Editors (ICMJE) criteria for authorship for this manuscript, take responsibility for the integrity of the work as a whole, and have given final approval for the version to be published.

Disclosures. Gunnar Kahlmeter, Jenny Åhman, and Erika Matuschek declare that they have no conflict of interest.

Compliance with ethics guidelines. All procedures followed were in accordance with the ethical standards of the responsible committee on human experimentation at the respective institutions and with the Helsinki Declaration of 1964, as revised in 2013. No patient identifiers other than sex and age were recorded and, as such, informed consent was not required for inclusion in this study.

Open Access. This article is distributed under the terms of the Creative Commons Attribution-NonCommercial 4.0 International License (http://creativecommons.org/licenses/ by-nc/4.0/), which permits any noncommercial use, distribution, and reproduction in any medium, provided you give appropriate credit to the original author(s) and the source, provide a link to the Creative Commons license, and indicate if changes were made.

\section{REFERENCES}

1. Allocati N, Masulli M, Alexeyev MF, Di Ilio C. Escherichia coli in Europe: an overview. Int J Environ Res Public Health. 2013;10:6235-54.

2. The Microbial Threat. Copenhagen: Invitational EU Conference, Denmark, September 1998 (Workshops 7-8 September).

3. Kahlmeter G. An international survey of the antimicrobial susceptibility of pathogens from acute uncomplicated urinary tract infections: the ECO.SENS project. J Antimicrob Chemother. 2003;51:69-76.

4. Kahlmeter G, Poulsen HO. Antimicrobial susceptibility of Escherichia coli from 
community-acquired urinary tract infections in Europe: the ECO.SENS study revisited. Int J Antimicrob Agents. 2012;39:45-51.

5. The European Committee on Antimicrobial Susceptibility Testing. EUCAST Disk Diffusion Test Manual. v 4.0; 2014. http://www.eucast.org.

6. The European Committee on Antimicrobial Susceptibility Testing. Breakpoint tables for interpretation of MICs and zone diameters. Version 4.0; 2014. http://www.eucast.org.

7. The Swedish Reference Group for Antibiotics (SRGA) and its subcommittee on antibiotics (SRGA-M). http://www.srga.org.

8. Lobet B, Valot A, Cattoir V, et al. Comparison of antimicrobial susceptibility of 1217 Escherichia coli isolates from women with hospital and community acquired urinary tract infections (Article in French). Presse Med. 2008;37:46-50.

9. Neuzillet V, Naber KG, Schito G, et al. French results of the ARESC study: clinical aspects and epidemiology of antimicrobial resistance in female patients with cystitis. Implications for therapy. Med Mal Infect. 2012;42:66-75.

10. Kresken M, Pfeifer Y, Hafner D, et al. Occurrence of multidrug resistance to oral antibiotics among Escherichia coli urine isolates from outpatient departments in Germany: extended-spectrum beta-lactamases and the role of fosfomycin. Int J Antimicrob Agents. 2014;44:295-300.

11. Schmiemann G, Gagyor I, Hummers-Pradier E, et al. Resistance profiles of urinary tract pathogens in general practice-an observational study. BMC Urol. 2012;21-33.
12. Garcia Garcia ML, Munoz Bellido JL, Garcia Rodriguez JA, et al. In vitro susceptibility of community-acquired urinary tract pathogens to commonly used antimicrobial agents in Spain: a comparative multicenter study (2002-2004). J Chemother. 2007;19:263-70.

13. Gobernado M, Valdes L, Alos JI, et al. Antimicrobial susceptibility of clinical Escherichia coli isolates from uncomplicated cystitis in women over a 1-year period in Spain. Rev Esp Quimoter. 2007;20:68-76.

14. Lindback H, Lindback J, Sylvan S, Melhus A. Low frequency of antibiotic resistance among urine isolates of Escherichia coli in the community, despite a major hospital outbreak with Klebsiella pneumoniae producing CTS-M-15 in Uppsala County. Scand J Infect Dis. 2010;42:243-8.

15. Bean DC, Krahe D, Wareham DW. Antimicrobial resistance in community and nosocomial Escherichia coli urinary tract isolates, London 2005-2006. Ann Clin Microbiol Antimicrob. $2008 ; 7: 13$.

16. Schito GC, Naber KG, Botto $H$, et al. The ARESC study: an international survey on the antimicrobial resistance of pathogens involved in uncomplicated urinary tract infections. Int J Antimicrob Agents. 2009;34:407-13.

17. Poulsen HO, Johansson A, Granholm S, Kahlmeter G, Sundqvist M. High genetic diversity of nitrofurantoin or mecillinam resistant Escherichia coli indicates low propensity for clonal spread. J Antimicrob Chemother. 2013;68:1974-7.

18. Theuretzbacher U, Van Bambeke F, Canton R, et al. Reviving old antibiotics. J Antimicrob Chemother. 2015;70(8):2177-81. 